\title{
BMJ Open Impact of Medicare prescription drug (Part D) coverage expansion on utilisation and financial burden of benzodiazepines among older adults: an interrupted time series analysis
}

\author{
Minghui Li (D) , ${ }^{1}$ Jing Yuan, ${ }^{2}$ Chelsea Dezfuli, ${ }^{1}$ Z Kevin Lu ${ }^{3}$
}

To cite: Li M, Yuan J, Dezfuli C, et al. Impact of Medicare prescription drug (Part D) coverage expansion on utilisation and financial burden of benzodiazepines among older adults: an interrupted time series analysis. BMJ Open 2021;11:e053717. doi:10.1136/ bmjopen-2021-053717

- Prepublication history for this paper is available online To view these files, please visit the journal online (http://dx.doi org/10.1136/bmjopen-2021053717).

$\mathrm{ML}$ and JY contributed equally.

Received 25 May 2021

Accepted 05 November 2021

Check for updates

(c) Author(s) (or their employer(s)) 2021. Re-use permitted under CC BY-NC. No commercial re-use. See rights and permissions. Published by BMJ.

For numbered affiliations see end of article.

Correspondence to

Dr Z Kevin Lu;

lu32@email.sc.edu

\section{ABSTRACT}

Objective Benzodiazepines were excluded from Medicare Part D coverage since its introduction in 2006. Part D expanded coverage for benzodiazepines in 2013. The objective was to examine the impact of Medicare Part D coverage expansion on the utilisation and financial burden of benzodiazepines in older adults.

Design Interrupted time series with a control group.

Setting Nationally representative sample.

Participants 53150468 users of benzodiazepines and 21749749 users of non-benzodiazepines (an alternative therapy) from the Medicare Current Beneficiary Survey between the pre-expansion (2006-2012) and postexpansion (2013-2017) periods.

Intervention Medicare Part D coverage expansion on benzodiazepines.

Primary and secondary outcome measures Annual rate of benzodiazepines and non-benzodiazepines, average number of benzodiazepines and non-benzodiazepines and average cost of benzodiazepines and nonbenzodiazepines.

Results After Medicare Part D coverage expansion, the level of the annual rate of benzodiazepines increased by $8.20 \%$ (95\% Cl: $6.07 \%$ to $10.32 \%)$ and the trend decreased by $1.03 \%$ each year $(95 \% \mathrm{Cl}$ : $-1.77 \%$ to $-0.29 \%$ ). The trend of the annual rate of non-benzodiazepines decreased by $0.72 \%$ each year (95\% Cl: $-1.11 \%$ to $-0.33 \%)$. For the average number of benzodiazepines, the level increased by 0.67 (95\% Cl: 0.52 to 0.82$)$ and the trend decreased by 0.10 each year ( $95 \% \mathrm{Cl}:-0.15$ to -0.05$)$. For the average number of non-benzodiazepines, the level decreased by $0.11(95 \%$ $\mathrm{Cl}:-0.21$ to -0.01 ) and the trend decreased by 0.04 each year ( $95 \% \mathrm{Cl}:-0.08$ to -0.01$)$. No significant level and trend changes were identified for the average cost of benzodiazepines and non-benzodiazepines.

Conclusions After Medicare Part D coverage expansion, there was a sudden increase in the utilisation of benzodiazepines and a decreasing trend in the long-term. The increase in the utilisation of benzodiazepines did not add a financial burden to older adults. As an alternative therapy, the utilisation of non-benzodiazepines decreased following the coverage expansion.
Strengths and limitations of this study

- The first study to examine the effects of Medicare Part D coverage expansion on benzodiazepines.

- Included both Part D claims and self-reported other prescription events from multiple payers.

- Used a quasi-experimental design of interrupted time series with a control group of those without Part D coverage.

- Non-Part D prescription events are self-reported which might not be complete.

\section{INTRODUCTION}

Medicare Part $\mathrm{D}$, the voluntary prescription drug benefit programme, was introduced in 2006 to alleviate some of the financial burdens of prescription drugs for Medicare beneficiaries. Certain classes of medications, however, were excluded from Part D coverage by the Medicare Prescription Drug, Improvement and Modernization Act. Standard Part D plans were not allowed to cover medications prescribed for anorexia, weight loss, weight gain, symptomatic relief of cough and colds, hair growth as well as barbiturates and benzodiazepines. ${ }^{1}$ Among the excluded medications, benzodiazepines are commonly used, especially in older adults. ${ }^{2}$ Benzodiazepines were excluded from the Part D coverage because of the increased risks of falls, hip fractures and worsened conditions like depression and urinary incontinence in older adults. ${ }^{3}$ However, this class of medications is a low cost and effective pharmacological treatment if used appropriately. To access benzodiazepines with the exclusion in place, Medicare beneficiaries need to have Part D plans with enhanced alternative coverage, have supplemental prescription drug coverage (such as Medicaid, Veterans Affairs or private insurance) or pay out-of-pocket. ${ }^{4}$ 
Since the start of Medicare Part D in 2006, the exclusion of benzodiazepine coverage has raised significant concerns. The coverage exclusion has limited patient access to and increased the financial burden of a widely used class of medications. ${ }^{5-7}$ The Medicare Improvement for Patients and Providers allowed Medicare to cover benzodiazepines when used for medically accepted indications starting in 2013. ${ }^{8}$

Benzodiazepines are a class of prescription medications that can provide rapid relief for many psychiatric disorders including anxiety and insomnia. ${ }^{910}$ This class is among the most commonly used psychiatric medications. ${ }^{11}$ Common benzodiazepines include alprazolam, clonazepam, diazepam, lorazepam and temazepam. Benzodiazepines are fast-acting and provide immediate relief. ${ }^{12}$ However, they have more risks when used long-term. ${ }^{13}$ Continued use of benzodiazepines can lead to dependence and worsening of symptoms. ${ }^{14}{ }^{15}$ The popular use of benzodiazepines is of concern in older adults because of the increased sensitivity. ${ }^{16}$ Non-benzodiazepines (Z-drugs) can be considered as an alternative treatment option in certain disease states when benzodiazepines are not available or not appropriate. ${ }^{17}$ This class is similar to benzodiazepines in terms of effectiveness, safety and cost. ${ }^{17} 18$ Common non-benzodiazepines include eszopiclone, zaleplon and zolpidem. Different from benzodiazepines, non-benzodiazepines were not excluded from Medicare Part D coverage.

After the introduction of Medicare Part D in 2006, when benzodiazepines were excluded from coverage, the utilisation of benzodiazepines was reduced but the utilisation of non-benzodiazepines was increased..$^{5-7}$ However, the impact of Medicare Part D coverage expansion in 2013 on the utilisation of benzodiazepines has yet to be determined. Although the rates of benzodiazepine prescriptions have been reported for years after 2013 in the literature, the overall trend of the utilisation of benzodiazepines remained unknown among older adults. ${ }^{19} 20$ No current literature has examined the impact of the benzodiazepine coverage expansion on the utilisation of non-benzodiazepines as an alternative therapy. In addition, there is currently no study assessing the financial burden of benzodiazepines and non-benzodiazepines before and after Medicare Part D coverage expansion. Therefore, to fill these gaps identified in the literature, this study aimed to examine the impact of Medicare Part D coverage expansion on the utilisation and financial burden of benzodiazepines and non-benzodiazepines (an alternative therapy) in older Medicare beneficiaries.

\section{METHODS}

\section{Data source}

This study used data from the Medicare Current Beneficiary Survey (MCBS) from 2006 to 2017. The MCBS is a multipurpose survey that is nationally representative of the Medicare population. Some goals of the MCBS are to determine sources of payment for all Medicare services, to trace health outcomes over time and to assess the impacts of Medicare programme changes. The MCBS was an appropriate data source for this study because both Medicare Part D prescription events and self-reported non-Part D prescription events were collected. It also collected detailed information on the source of payments from Medicare, beneficiary out-of-pocket and supplemental insurance.

\section{Study design}

This study used the quasi-experimental design of interrupted time series. The trend of the utilisation and financial burden of benzodiazepines and non-benzodiazepines were plotted and compared before and after the coverage expansion. Beginning in 2013, benzodiazepines were covered under Medicare Part D. The pre-expansion period was 2006-2012 and the post-expansion period was 2013-2017 in this study.

\section{Control group}

The treatment group was composed of Medicare beneficiaries aged 65 years or older with Part D coverage; while the control group was composed of Medicare beneficiaries aged 65 years or older without Part D coverage. The benzodiazepine coverage expansion has an impact on individuals in the treatment group but no impact on those in the control group. The inclusion of the control group could reduce the threat to internal validity due to history.

\section{Alternative treatment}

Non-benzodiazepines can be considered as an alternative treatment option when benzodiazepines are not available or not appropriate. During the whole study period, non-benzodiazepines are covered under Medicare Part D. In the pre-expansion period, when benzodiazepines were excluded from Medicare Part D coverage, clinicians could prescribe non-benzodiazepines as an alternative therapy for patients with no access to benzodiazepines. In the post-expansion period, both benzodiazepines and non-benzodiazepines were covered under Medicare Part D.

\section{Measurement}

Prescriptions of benzodiazepines and nonbenzodiazepines were measured by Medicare Part D prescription events and self-reported non-Part D prescription events. The total prescription costs were categorised into Medicare costs, out-of-pocket costs and other costs. Medicare administrative data were used to determine Part $\mathrm{D}$ coverage. The rate and average number were used to indicate the utilisation and the average cost was used to indicate the financial burden of benzodiazepines and non-benzodiazepines. The annual prescription rate was measured by the total number of individuals who had any prescriptions divided by the total number of individuals in a given year. The annual average prescription number was measured by the total number of prescriptions divided by the total number of individuals in a given 


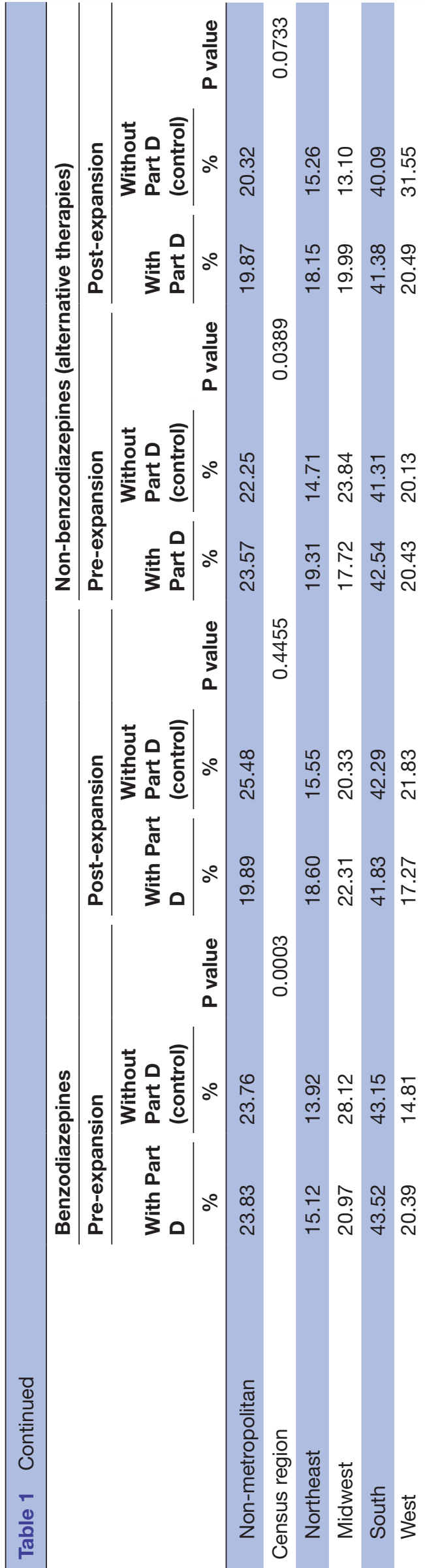

year. The annual average prescription cost was measured by the total prescription costs divided by the total number of individuals in a given year.

\section{Statistical analysis}

Demographic and socioeconomic characteristics of users of benzodiazepines and non-benzodiazepines during the pre-expansion and post-expansion periods were compared between those with and without Medicare Part D coverage using $\chi^{2}$ tests. Segmented regression analyses were used in the interrupted time series design to examine the impact of Medicare Part D coverage expansion. The annual rate, average number and average cost of benzodiazepines and non-benzodiazepines were plotted in graphs. Regression lines were fitted and compared between the pre-expansion and post-expansion periods. Both the level change and trend change (slope change) were estimated to examine the immediate and sustained effects of the coverage expansion. Survey sampling weights were applied to generate national estimates.

\section{Patient and public involvement}

Patients and/or the public were not involved in the design, or conduct, or reporting, or dissemination plans of this research. The lack of involvement is compliant with the data protection laws.

\section{RESULTS}

This study included 53150468 older adults who used benzodiazepines and 21749749 older adults who used non-benzodiazepines. The majority of users of benzodiazepines and non-benzodiazepines were aged 65-74 years, women and non-Hispanic white. Among users of benzodiazepines, $27077542(50.95 \%)$ were included in the preexpansion period and 26072926 (49.05\%) were included in the post-expansion period. Those with Part D coverage were significantly different from those without Part D coverage in gender, race, education, marital status and income (table 1). Among users of non-benzodiazepines, $13979845(64.28 \%)$ were included in the pre-expansion period and $7769904(35.72 \%)$ were included in the postexpansion period. The distributions of age, residence and census region were similar between those with and without Part D coverage (table 1).

\section{Annual rate}

For the annual rate of benzodiazepines, the trend was stable during the pre-expansion period. After the coverage expansion, the rate increased suddenly from $10.14 \%$ in 2012 to $16.86 \%$ in 2013. During the postexpansion period, there was a decreasing trend. In the control group, the trend was stable during the whole study period (figure 1). After including the control group, the level increased by $8.20 \%$ (95\% CI: $6.07 \%$ to $10.32 \%$ ) in 2013 and the trend decreased by $1.03 \%$ each year (95\% CI: $-1.77 \%$ to $-0.29 \%$ ) (table 2). For different types of prescription events, Part D events accounted for 

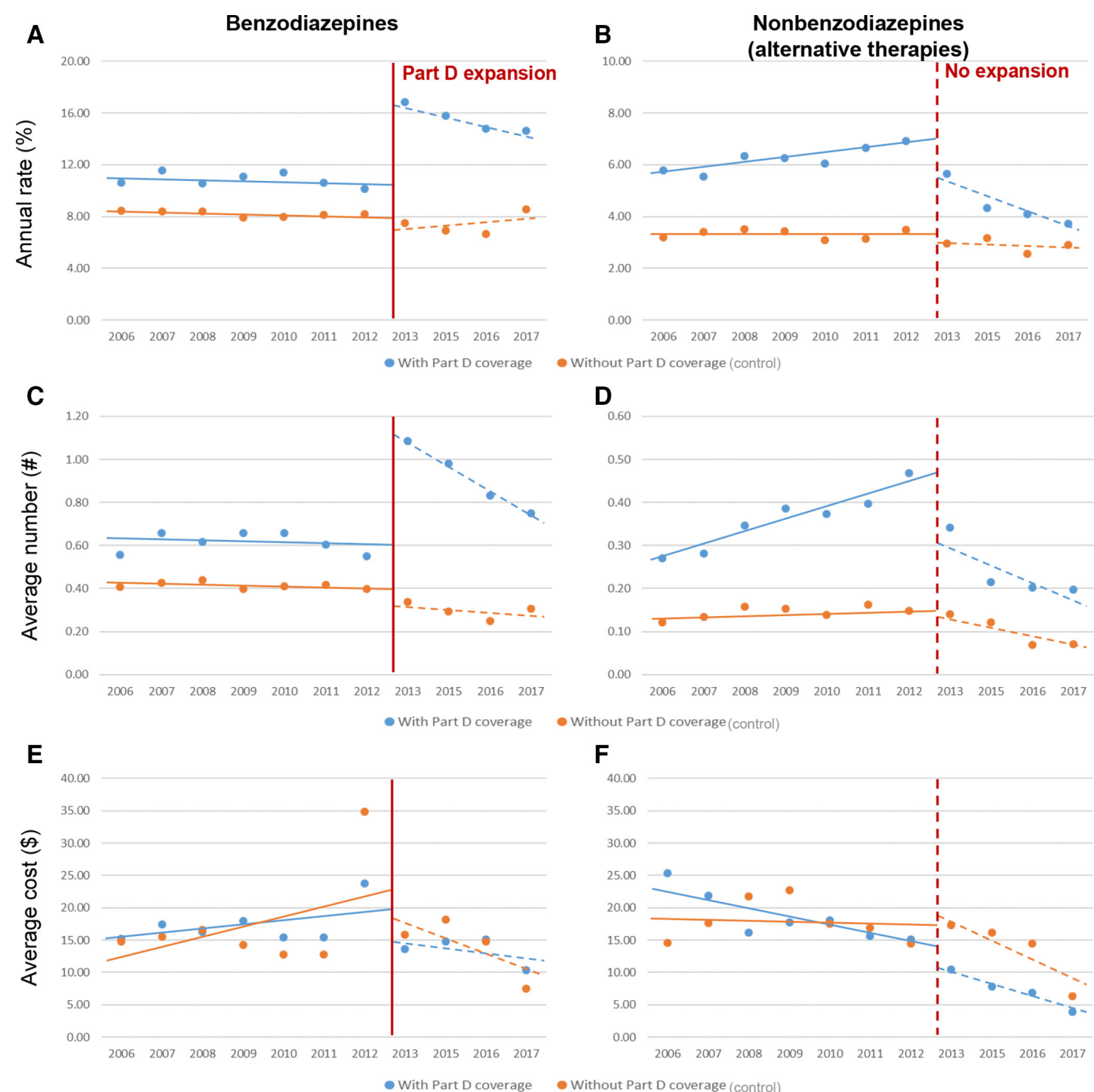

Figure 1 Change in the annual rate, average number and average cost of benzodiazepines and non-benzodiazepines before and after Medicare Part D coverage expansion. (A) Annual rate of benzodiazepines; (B) annual rate of non-benzodiazepines; (C) average number of benzodiazepines; (D) average number of non-benzodiazepines; (E) average cost of benzodiazepines; $(F)$ average cost of non-benzodiazepines.

a small proportion during the pre-expansion period but a large proportion during the post-expansion period (figure 2). The level of Part D events increased by $14.62 \%$ (95\% CI: $12.50 \%$ to $16.73 \%$ ) in 2013 , the trend of Part D events decreased by $1.10 \%$ each year (95\% CI: $-1.83 \%$ to $-0.37 \%$ ), and the level of non-Part $\mathrm{D}$ events decreased by $5.08 \%$ ( $95 \%$ CI: $-6.89 \%$ to $-3.27 \%$ ) in 2013 (table 3 ).

When assessing the annual rate of non-benzodiazepines, there was an increasing trend during the pre-expansion period. After the coverage expansion, there was a reduction in the rate from $6.93 \%$ in 2012 to $5.64 \%$ in 2013 . There was a decreasing trend during the post-expansion period. In the control group, the trend was stable during the whole study period (figure 1). After including the control group, the trend decreased by $0.72 \%$ each year (95\% CI: $-1.11 \%$ to $-0.33 \%$ ) (table 2). For different types of prescription events, Part D events accounted for a large proportion during the whole study period (figure 2).
The trend of Part D events decreased by $0.77 \%$ each year (95\% CI: $-1.18 \%$ to $-0.37 \%$ ) (table 3 ).

\section{Average number}

For the annual average number of benzodiazepines, the trend was stable during the pre-expansion period. After the coverage expansion, the average number increased suddenly from 0.55 in 2012 to 1.09 in 2013. During the post-expansion period, there was a decreasing trend. In the control group, the trend was stable during the whole study period (figure 1). After including the control group, the level increased by 0.67 (95\% CI: 0.52 to 0.82 ) in 2013 and the trend decreased by 0.10 each year $(95 \%$ CI: -0.15 to -0.05) (table 1). For different types of prescription events, Part D events accounted for a small proportion during the pre-expansion period but a large proportion during the post-expansion period (figure 2). The level of Part D events increased by 0.77 (95\% CI: 0.66 to 0.87 ) in 
Table 2 Change in the annual rate, average number and average cost of benzodiazepines and non-benzodiazepines before and after Medicare Part D coverage expansion

\begin{tabular}{|c|c|c|c|c|c|c|}
\hline & \multicolumn{2}{|c|}{ Annual rate } & \multicolumn{2}{|c|}{ Average number } & \multicolumn{2}{|c|}{ Average cost } \\
\hline & $\beta$ & $95 \% \mathrm{Cl}$ & $\boldsymbol{\beta}$ & $95 \% \mathrm{Cl}$ & $\beta$ & $95 \% \mathrm{Cl}$ \\
\hline \multicolumn{7}{|l|}{ Benzodiazepines } \\
\hline Intercept & 8.44 & 7.54 to 9.35 & 0.42 & 0.36 to 0.49 & 10.12 & 1.10 to 19.13 \\
\hline Year & -0.06 & -0.26 to 0.14 & 0.00 & -0.02 to 0.01 & 1.82 & -0.20 to 3.83 \\
\hline Expansion & -1.34 & -2.85 to 0.16 & -0.07 & -0.18 to 0.03 & -1.59 & -16.55 to 13.36 \\
\hline Year after expansion & 0.35 & -0.17 to 0.87 & -0.01 & -0.05 to 0.03 & -4.68 & -9.86 to 0.50 \\
\hline Part D & 2.76 & 1.47 to 4.04 & 0.20 & 0.11 to 0.30 & 4.29 & -8.47 to 17.04 \\
\hline Year $\times$ Part D & -0.03 & -0.31 to 0.26 & 0.00 & -0.02 to 0.02 & -1.07 & -3.93 to 1.78 \\
\hline Expansion ×Part D (level change) & 8.20 & 6.07 to 10.32 & 0.67 & 0.52 to 0.82 & -2.09 & -23.24 to 19.06 \\
\hline $\begin{array}{l}\text { Year after expansion } \times \text { Part D (trend } \\
\text { change) }\end{array}$ & -1.03 & -1.77 to 0.29 & -0.10 & -0.15 to 0.05 & 2.97 & -4.36 to 10.29 \\
\hline \multicolumn{7}{|c|}{ Non-benzodiazepines (alternative therapies) } \\
\hline Intercept & 3.34 & 2.85 to 3.82 & 0.13 & 0.09 to 0.17 & 18.79 & 13.96 to 23.63 \\
\hline Year & 0.00 & -0.11 to 0.11 & 0.00 & -0.01 to 0.01 & -0.21 & -1.30 to 0.87 \\
\hline Expansion & -0.23 & -1.03 to 0.57 & 0.01 & -0.06 to 0.08 & 4.97 & -3.04 to 12.99 \\
\hline Year after expansion & -0.07 & -0.35 to 0.20 & -0.03 & -0.06 to 0.08 & -3.25 & -6.02 to 0.47 \\
\hline Part D & 2.13 & 1.44 to 2.81 & 0.11 & 0.05 to 0.17 & 5.67 & -1.17 to 12.50 \\
\hline Year $\times$ Part D & 0.19 & 0.04 to 0.35 & 0.03 & 0.01 to 0.04 & -1.26 & -2.78 to 0.27 \\
\hline Expansion $\times$ Part D (level change) & -0.61 & -1.75 to 0.52 & -0.11 & -0.21 to 0.01 & -6.73 & -18.07 to 4.60 \\
\hline $\begin{array}{l}\text { Year after expansion } \times \text { Part } D \text { (trend } \\
\text { change) }\end{array}$ & -0.72 & -1.11 to 0.33 & -0.04 & -0.08 to 0.01 & 2.66 & -1.27 to 6.59 \\
\hline
\end{tabular}

2013 and the trend decreased by 0.04 each year $(95 \% \mathrm{CI}$ : -0.08 to -0.01 ) (table 2 ).

When assessing the annual average number of nonbenzodiazepines, there was an increasing trend during the pre-expansion period. After the coverage expansion, there was a reduction in the average number from 0.47 in 2012 to 0.34 in 2013. There was a decreasing trend during the post-expansion period. In the control group, the trend was stable during the whole study period (figure 1). After including the control group, the level decreased by 0.11 (95\% CI: -0.21 to -0.01 ) in 2013 and the trend decreased by 0.04 each year (95\% CI: -0.08 to -0.01 ) (table 1 ). For different types of prescription events, Part D events accounted for a large proportion during the whole study period (figure 2). No significant level and trend changes were identified in Part D and non-Part D events (table 2).

\section{Average cost}

For the annual average cost of benzodiazepines and nonbenzodiazepines, the trends were stable during the whole study period, except for some outliers. Similar trends were observed in the control groups (figure 1). No significant level and trend changes were identified after including the control groups (table 1). For different sources of payment, the major source was Medicare followed by beneficiary out-of-pocket and others, during the whole study period (figure 2). No significant level and trend changes were identified in Medicare, out-of-pocket and other costs (table 2).

\section{DISCUSSIONS}

Medicare Part D coverage expansion increased the utilisation of benzodiazepines. From 2012 to 2013, the rate increased by $8.20 \%$ and the average number increased by 0.67 , driven by the increase in Part $\mathrm{D}$ events. However, the increasing effect did not last long. After the coverage expansion, the rate decreased by $1.03 \%$ and the average number decreased by 0.10 each year, driven by the reduction in Part D events. Even though the long-term trend is decreasing, the utilisation of benzodiazepines in the post-expansion period was still higher than that in the pre-expansion period. The immediate increasing effect found in this study is similar to previous studies. Albrecht et al found that among older adults with insomnia, the prescription rate of benzodiazepines increased from $1.1 \%$ in 2012 to $17.6 \%$ in $2013 .{ }^{19}$ Maust et al found that among older Medicare Advantage enrollees, there was a sudden increase in the monthly proportions of benzodiazepines from lower than $1 \%$ in 2012 to around $6 \%$ in $2013 .{ }^{20}$ The sustained decreasing effect found in this study is different from previous studies. Maust $e t$ al found that there was a stable trend in the monthly proportions of benzodiazepines after 2013 among older adults enrolled in Medicare 

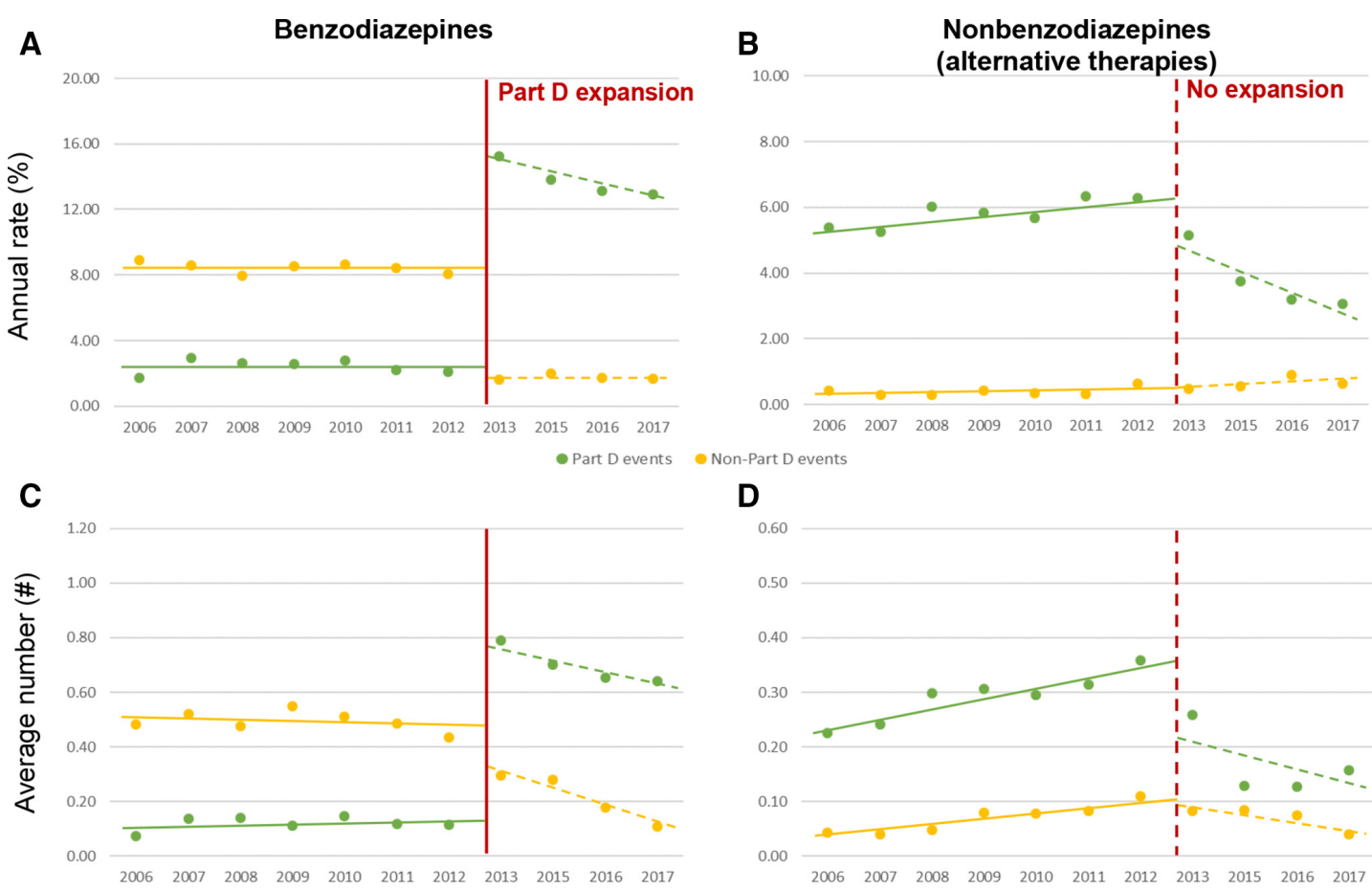

D
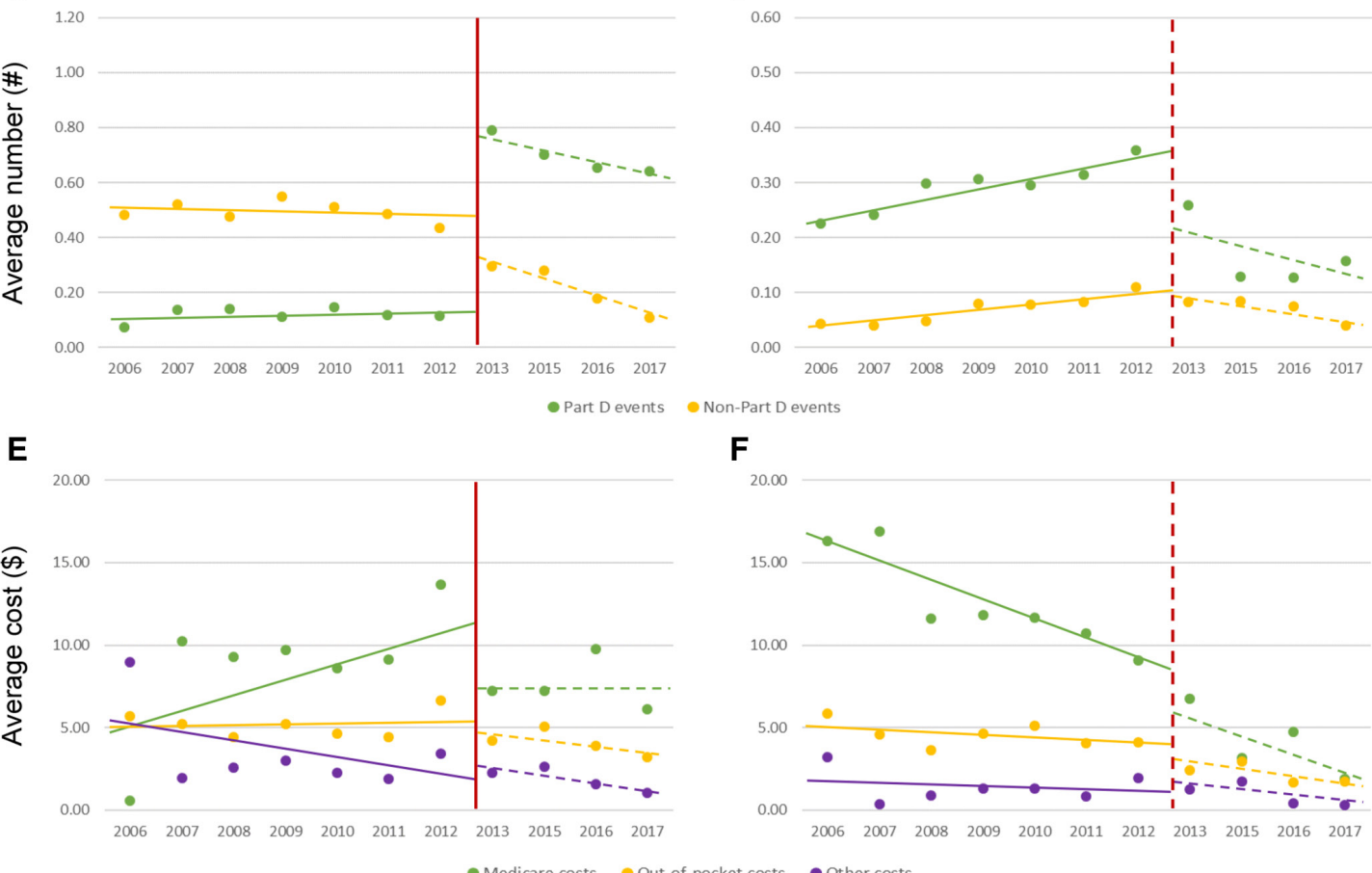

$\mathbf{F}$

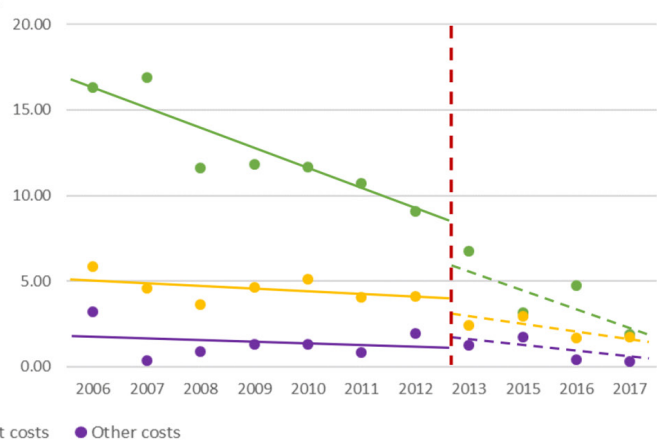

Figure 2 Change in the annual rate, average number and average cost of benzodiazepines and non-benzodiazepines before and after Medicare Part D coverage expansion by different sources. (A) Annual rate of benzodiazepines; (B) annual rate of nonbenzodiazepines; (C) average number of benzodiazepines; (D) average number of non-benzodiazepines; (E) average cost of benzodiazepines; (F) average cost of non-benzodiazepines.

Advantage plans. ${ }^{20}$ This might be because of differences in study populations. This study included older adults with Medicare Advantage Part D or stand-alone Part D plans. In addition, this study identified a transition in the types of prescription events from non-Part D events to Part $\mathrm{D}$ events following the coverage expansion.

The coverage expansion did not increase the financial burden of benzodiazepines among older adults. After the coverage expansion, the annual average cost of benzodiazepines did not change in both short and long terms and by different sources of payment. Given the increase in the utilisation of benzodiazepines, the cost of each prescription is lower in the post-expansion period. This might be because more generic drugs are used under Medicare. To our knowledge, this study is the first to examine the impact of Medicare Part D coverage expansion on the financial burden of benzodiazepines.
The coverage expansion reduced the utilisation of non-benzodiazepines. From 2012 to 2013, the average number decreased by 0.11 . After the coverage expansion, the rate decreased by 0.07 and the average number decreased by 0.04 each year. Because benzodiazepines are covered under Medicare Part D after the coverage expansion, non-benzodiazepines are less likely to be used as an alternative therapy. In addition, the coverage expansion has no impact on the financial burden of non-benzodiazepines. As far as we know, this is the first study to examine the impact of Medicare Part D coverage expansion on non-benzodiazepines.

Through the expansion of Part D coverage, the inappropriate use, misuse and abuse of benzodiazepines might increase. This is a special concern in older adults because of the increased risk of cognitive impairment, delirium, falls, fractures and motor vehicle crashes. ${ }^{21}$ 
Table 3 Change in the annual rate, average number and average cost of benzodiazepines and non-benzodiazepines before and after Medicare Part D coverage expansion by different sources

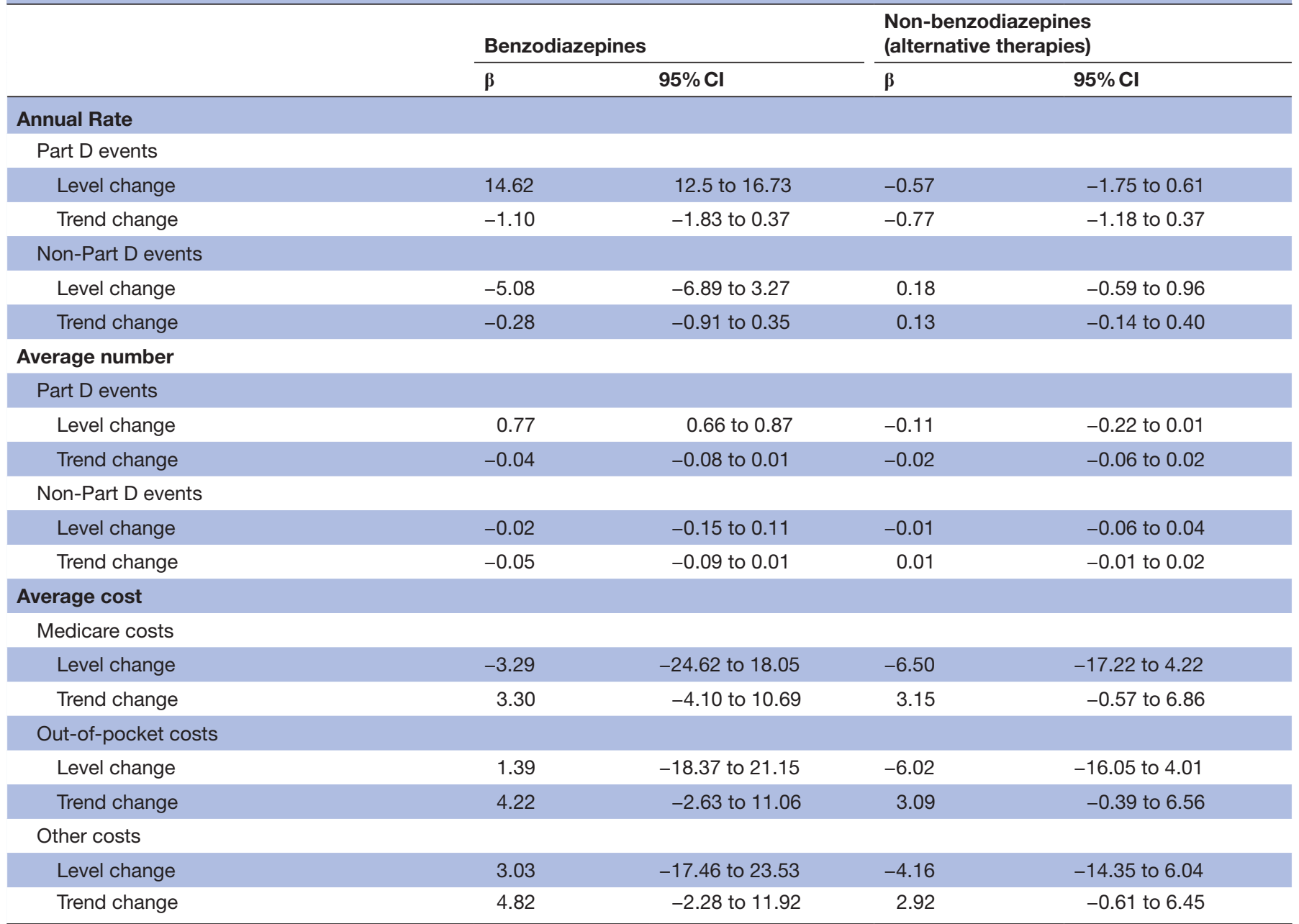

After the coverage expansion, Maust et al found that the rates of overdose and fall-related injury increased significantly in adults 80 years and older. ${ }^{20}$ In older adults, the use of benzodiazepines might exacerbate certain disease states like dementia, a history of falls and kidney disease. ${ }^{22}$ Concurrent use of benzodiazepines with opioids and central nervous system agents could increase the risk of overdose, falls and fracture in older adults. ${ }^{23}$

Both healthcare providers and patients play active roles in the appropriate use of benzodiazepines. Physicians and other prescribers should involve patients in the discussion on the benefits and risks of using benzodiazepines and the possibility of inappropriate use, misuse and abuse. Special attention should be paid to educate patients on the safety of long-term use of benzodiazepines. Pharmacists can provide medication therapy management services to help older adults better use benzodiazepines. During the consultation, pharmacists should review potential drug-disease and drug-drug interactions of benzodiazepines and recommend deprescribing if necessary. The coverage expansion increased the utilisation of benzodiazepines in older adults with no additional financial burden. However, inappropriate use, misuse and abuse of benzodiazepines are associated with higher healthcare utilisation and financial burden in the long term. Patients should be actively involved in clinical decision-making and exploring non-pharmacological and alternative treatments to benzodiazepines.

The goal of Part D coverage expansion was to alleviate some of the financial burdens Medicare beneficiaries might have faced in accessing and using benzodiazepines. The coverage expansion led to an increase in access to and utilisation of benzodiazepines. The financial burden of benzodiazepines remained unchanged. However, the coverage expansion led to a reduction in the utilisation of non-benzodiazepines. It is of great significance to consider the clinical consequences and economic impact of a policy change. This study could inform decisionmakers at the Centers for Medicare and Medicaid Services on future policy decisions in the expansion of Part D coverage to other excluded medications. For example, weight loss medications could reduce the risk of diabetes and generate cost savings, but are excluded from Part D coverage. ${ }^{24}$ There are clinical and economic benefits to cover weight loss prescriptions under Medicare Part D. A comprehensive policy evaluation like this study is 
warranted to consider potential coverage expansion on other excluded medications.

\section{Limitations}

Some limitations should be considered when interpreting the study findings. First, non-Part D prescription events are self-reported which might not be complete. Respondents of the MCBS are asked to save healthcare-related paperwork and provide medication bottles, containers or bags during the interview. Thus, the impact should be minimal. Second, the MCBS data in 2014 are not released because of sampling and data collection issues. The MCBS has been continuously conducted for over 25 years. Even though the data in 2014 is not available, this study is still able to examine the long-term effect of the coverage expansion by including years after that. Third, the control group of older Medicare beneficiaries without Part D coverage might not be the best. The control group is not subject to the coverage expansion. However, it is not comparable to the treatment group in all aspects.

\section{CONCLUSIONS}

After Medicare Part D coverage expansion, there was a sudden increase in the utilisation of benzodiazepines and a decreasing trend in the long term. The increase in the utilisation of benzodiazepines did not add a financial burden to older adults. As an alternative therapy, the utilisation of non-benzodiazepines decreased following the coverage expansion. Given the increase in the utilisation of benzodiazepines, healthcare providers and patients should minimise the potential for inappropriate use, misuse and abuse. For future policy decisions, both the clinical consequences and economic impact should be carefully evaluated. This study could inform the Centers for Medicare and Medicaid Services to make Part D coverage decisions on other excluded medications.

\section{Author affiliations}

${ }^{1}$ Department of Clinical Pharmacy and Translational Science, The University of Tennessee Health Science Center, Memphis, Tennessee, USA

${ }^{2}$ Department of Clinical Pharmacy and Pharmacy Administration, Fudan University, Shanghai, China

${ }^{3}$ Department of Clinical Pharmacy and Outcomes Sciences, University of South Carolina, Columbia, Columbia, USA

Contributors ML contributed to the conceptualisation, study design, data acquisition, data management, statistical analysis and manuscript writing. JY contributed to the conceptualisation, study design, data interpretation and manuscript writing. $C D$ contributed to the literature review and manuscript writing. $\mathrm{KL}$ contributed to the conceptualisation, study design and supervision. All authors revised the manuscript and approved the final version of the manuscript. $\mathrm{KL}$ is the guarantor of this study.

Funding The authors have not declared a specific grant for this research from any funding agency in the public, commercial or not-for-profit sectors.

Competing interests None declared.

Patient and public involvement Patients and/or the public were not involved in the design, or conduct, or reporting, or dissemination plans of this research.

Patient consent for publication Not applicable.

Ethics approval This study was approved by the University of Tennessee Health Science Center Institutional Review Board (21-08174-NHSR). Data were anonymised before the authors accessed them for the purpose of this study.
Provenance and peer review Not commissioned; externally peer reviewed.

Data availability statement Data may be obtained from a third party and are not publicly available. No data are available. The Medicare Current Beneficiary Survey is maintained by the Centers for Medicare and Medicaid Services and contains identified data. These data can be accessed only by certified personnel.

Open access This is an open access article distributed in accordance with the Creative Commons Attribution Non Commercial (CC BY-NC 4.0) license, which permits others to distribute, remix, adapt, build upon this work non-commercially, and license their derivative works on different terms, provided the original work is properly cited, appropriate credit is given, any changes made indicated, and the use is non-commercial. See: http://creativecommons.org/licenses/by-nc/4.0/.

ORCID iD

Minghui Li http://orcid.org/0000-0003-1909-6260

\section{REFERENCES}

1 Hanlon JT, Schmader KE. Essentials of prescribing in the era of the medicare prescription drug improvement and modernization act. $A m$ $J$ Geriatr Pharmacother 2005;3:123-6.

2 Olfson M, King M, Schoenbaum M. Benzodiazepine use in the United States. JAMA Psychiatry 2015;72:136-42.

3 Fick DM, Cooper JW, Wade WE, et al. Updating the Beers criteria for potentially inappropriate medication use in older adults: results of a US consensus panel of experts. Arch Intern Med 2003;163:2716-24.

4 Bambauer KZ, Sabin JE, Soumerai SB. The exclusion of benzodiazepine coverage in medicare: simple steps for avoiding a public health crisis. Psychiatr Serv 2005;56:1143-6.

5 Chen H, Nwangwu A, Aparasu R, et al. The impact of medicare part D on psychotropic utilization and financial burden for communitybased seniors. Psychiatr Serv 2008;59:1191-7.

6 Ong MK, Xu H, Zhang L, et al. Effect of medicare part D benzodiazepine exclusion on psychotropic use in benzodiazepine users. J Am Geriatr Soc 2012;60:1292-7.

7 Stubbings J, Lau DT. Medicare part D research highlights and policy updates, 2013: impact and insights. Clin Ther 2013;35:402-12.

8 LeMasurier JD, Edgar B. MIPPA: first broad changes to Medicare Part D plan operations. Am Health Drug Benefits 2009;2:111-8.

9 Cloos J-M, Ferreira V. Current use of benzodiazepines in anxiety disorders. Curr Opin Psychiatry 2009;22:90-5.

10 Bastien CH, LeBlanc M, Carrier J, et al. Sleep EEG power spectra, insomnia, and chronic use of benzodiazepines. Sleep 2003;26:313-7.

11 Moore TJ, Mattison DR. Adult utilization of psychiatric drugs and differences by sex, age, and race. JAMA Intern Med 2017;177:274-5.

12 Griffin CE, Kaye AM, Bueno FR, et al. Benzodiazepine pharmacology and central nervous system-mediated effects. Ochsner $J$ 2013;13:214-23.

13 Schmitz A. Benzodiazepine use, misuse, and abuse: a review. Ment Health Clin 2016;6:120-6.

14 Votaw VR, Geyer R, Rieselbach MM, et al. The epidemiology of benzodiazepine misuse: a systematic review. Drug Alcohol Depend 2019;200:95-114.

15 Jeffreys M, Capehart B, Friedman MJ. Pharmacotherapy for posttraumatic stress disorder: review with clinical applications. $J$ Rehabil Res Dev 2012;49:703-15.

16 Bogunovic OJ, Greenfield SF. Practical geriatrics: use of benzodiazepines among elderly patients. Psychiatr Serv 2004;55:233-5.

17 Schroeck JL, Ford J, Conway EL, et al. Review of safety and efficacy of sleep medicines in older adults. Clin Ther 2016;38:2340-72.

18 Dündar Y, Boland A, Strobl J, et al. Newer hypnotic drugs for the short-term management of insomnia: a systematic review and economic evaluation. Health Technol Assess 2004;8:iii-x, 1-125.

19 Albrecht B, Staiger PK, Hall K, et al. Benzodiazepine use and aggressive behaviour: a systematic review. Aust N Z J Psychiatry 2014;48:1096-114.

20 Maust DT, Kales HC, Wiechers IR, et al. No end in sight: benzodiazepine use in older adults in the United States. J Am Geriatr Soc 2016;64:2546-53.

212019 American Geriatrics Society Beers Criteria $®$ Update Expert Panel. American geriatrics Society 2019 updated AGS beers Criteria $₫$ for potentially inappropriate medication use in older adults. J Am Geriatr Soc 2019;67:674-94.

22 Hanlon JT, Semla TP, Schmader KE. Alternative medications for medications in the use of high-risk medications in the elderly and potentially harmful drug-disease interactions in the elderly quality measures. J Am Geriatr Soc 2015;63:e8-18. 
23 Hines LE, Murphy JE. Potentially harmful drug-drug interactions in the elderly: a review. Am J Geriatr Pharmacother 2011;9:364-77.
24 Thorpe KE, Yang Z, Long KM, et al. The impact of weight loss among seniors on Medicare spending. Health Econ Rev 2013;3:7. 